\title{
A Brief Review of Materials and Designs for Homemade Masks to Protect Against COVID-19
}

\author{
Jago Strong-Wright \\ June 2020 \\ University of Cambridge \\ COVID-19 - SARS-CoV-2 - Masks - Materials - Pandemic
}

\section{ACKNOWLEDGEMENTS}

Many thanks to Professor John Leake for his guidance and advice, and to Professor James Elliott and Professor Serena Best for their support and encouragement.

Abstract - It is highly likely that the wearing of face masks reduces the rate of respiratory infections (e.g. SARS-CoV-2), to protect both the user and those around them. This paper sets out to review the areas that effect the efficacy of masks, the materials, design, hygiene and fit testing, in order to make recommendations as to how to make mask from resources found in most homes for when commercial models are unavailable.

This paper finds that a mask constructed with a filter made from high thread count cotton is likely to provide a reasonable level of protection $(\sim 70 \%$ filtration) and that if a layers of other materials such as chiffon or silk is added the filtration may be much higher $(\geq 90 \%)$. There is also some promise in less available materials such as vacuum cleaner bags and air conditioner filters. Examples of fabric and rigid designs are reviewed but most are limited by the fit to the users which is hard to determine in a home setting.

It would be extremely helpful if a method was devised for people to test or be tested for the fit of home made masks. In the mean time careful thought should be given to whether the user judges a good fit. Users should also be careful to practice other means of hygiene and distancing.

\section{INTRODUCTION}

The wearing of face masks while in contact with other people is likely to significantly reduce the spread of SARS-CoV-2 and other respiratory viruses [1][2][3][4][5][6][7][8][9][10]. In an ideal world every person would have access to certified masks but clearly this is unrealistic during a pandemic. It is therefore clear that alternatives are required but these alternatives need to be suited to their purpose. There is little clarity on what is required to satisfy this and so this review seeks to shine a light on what little evidence there is.

\section{CRITERIA}

The masks currently being employed in hospitals in high risk situations (in the UK) are filtering respirators and in lower risk situations fluid replant surgical masks [11]. This is a lower standard than is usually used when shortages are not an issue[12], even at the start of the pandemic before the disease was endemic in the UK it was recommended to use filtering respirators for all patient contact[13]. It therefore seems sensible to aspire to the requirements for a filtering face piece respirator. A summary of the necessary requirements derived from EN 149 [14] are:

1) The mask should have a total minimum inward leakage of $2 \%$ with the filter material penetrated by less than $1 \%$ when a sample is tested (for particles of a size between 0.02 and $2 \mu \mathrm{m})$.

2) The mask should not collect air such that it allows a build up of carbon dioxide.

3) The maximum resistance to breathing should be less than 3 mbar.

4) The mask should still satisfy these criteria while exercising and going about normal activities.

Similar criteria exist in the United States where masks are certified to be "N95"[15].

Some criteria related to the durability and industry specific needs (e.g. flammability) are not included here as we are looking to replicate the functionality of the mask with easily accessible materials but not to create a certifiable device. 
Many studies do not test material with particles of a particular size, instead they test with a variety of viruses and bacteria. Since these are the particles that need to be filtered out it is reasonable to look for the filtration efficiency for viruses instead of particular particle sizes.

The standards also require that the fit of the mask be tested for each user (fit tested). This can be conducted qualitatively whereby the user wears the mask and a hood into which a flavoured aerosol is sprayed and the user judges if they can taste it or not, or quantitatively where the particulate level at particular sizes is measured inside and outside the mask [16].

These criteria provide a good benchmark for this review to compare possible solutions to. There is some evidence to suggest that proper respirators are no more effective at reducing infection rate than surgical mask [17]. The subjects in this study had a very low rate of compliance mainly stating that they found masks uncomfortable, the compliance was also lower in the respirator arm of the trial. Therefore, it will be important to emphasise comfort and wear-ability when considering mask designs.

\section{MATERIALS}

From the criteria detailed above it is clear that one of the key factors in finding an efficient masks is in the material they are made from. The focus will be on commonly available materials, with preference to ones already present in most houses. As can be seen from the following analysis there are few studies useful for this review.

\section{A. Testing the Efficacy of Homemade Masks:} Would They Protect in an Influenza Pandemic? [18]

1) Authors: Anna Davies, Katy-Anne Thompson, Karthika Giri, George Kafatos, Jimmy Walker, and Allan Bennett, 2013

2) Overview: This paper analysed a variety of household materials for their ability to block Bacillus atrophaeus and Bacteriophage MS2 directly and by getting volunteers to make masks out of them and testing their fit. They found a significantly lower fit factor in home made masks than surgical masks but a significant reduction in the amount of bacteria expelled by volunteers when they coughed. There was little specification of the design of the home made masks.

The useful part of this paper is their results of the direct material measurements. The paper tested a variety of materials and found that the most effective of their selection at filtering the virus sample were surgical masks $(90 \pm 3 \%$ filtration), vacuum cleaner bags $(86 \pm 2 \%)$, tea towel $(70 \pm 23 \%)$, "cotton mix" (70.24 $\pm 0.08 \%)$ and antimicrobial pillowcases $(70 \pm 7 \%)$. Although when discussed in the media it is commonly suggested that the paper tested the samples with multiple layers this is somewhat misleading as they only used multiple layers when testing with the bacteria sample, although it is somewhat useful to acknowledge that multiple layers are likely to increase the filtration efficiency.

The paper also measured that pressure drop across the materials and found that the materials all had a similar pressure drop as the surgical mask except the vacuum cleaner bag which had about twice the pressure drop. The paper concludes that the vacuum cleaner bag has too high a pressure drop to be useful and was also too stiff but it could still be useful for some designs.

3) Discussion and take away: Although this study is some of the only qualitative measurements of different materials efficiency this paper is of limited help fullness due to the lack of specificity about the materials. When I contacted the author they reported that as it was a provisional study they didn't have a record of the brands or specifications of the materials. Some sources would suggest that the tea towel was likely to be made of cotton, possibly with some polyester [19][20][21], and that pillow cases are also usually a high thread count cotton [22][23]. Additionally there is a small amount of other research that supports the filtration efficiency of vacuum cleaner bags [24] although this again does not name the brands.

While the author did not record the brand of the vacuum cleaner bags most are sold as having some filtering qualities (although these are unverifiable)[25][26].

B. Aerosol Filtration Efficiency of Common Fabrics Used in Respiratory Cloth Masks[27]

1) Authors: Abhiteja Konda, Abhinav Prakash, Gregory A. Moss, Michael Schmoldt, Gregory D. 


\begin{tabular}{|c|c|c|}
\hline \multirow{2}{*}{ Sample/fabric } & \multicolumn{2}{|c|}{ Filter efficiency $/ \%$} \\
\hline & $<300 \mathrm{~nm}$ & $\geq 300 \mathrm{~nm}$ \\
\hline N95 & $85 \pm 15$ & $99.9 \pm 0.1$ \\
\hline Surgical mask & $76 \pm 22$ & $99.6 \pm 0.1$ \\
\hline Cotton (600TPI), 1 layer & $79 \pm 23$ & $98.4 \pm 0.2$ \\
\hline Cotton (600TPI), 2 layer & $82 \pm 19$ & $99.5 \pm 0.1$ \\
\hline $\begin{array}{l}\text { Chiffon }(90 \% \text { polyester, } 10 \% \\
\text { spandex), } 1 \text { layer }\end{array}$ & $67 \pm 16$ & $73 \pm 2$ \\
\hline $\begin{array}{l}\text { Chiffon ( } 90 \% \text { polyester, } 10 \% \\
\text { spandex), } 2 \text { layers }\end{array}$ & $83 \pm 9$ & $90 \pm 1$ \\
\hline Natural Silk, 2 layers & $65 \pm 10$ & $65 \pm 2$ \\
\hline Natural Silk, 4 layers & $86 \pm 5$ & $88 \pm 1$ \\
\hline $\begin{array}{l}\text { Cotton (600TPI), } 1 \text { layer and } \\
\text { Chiffon, } 2 \text { layers }\end{array}$ & $97 \pm 1$ & $99.1 \pm 0.2$ \\
\hline $\begin{array}{l}\text { Cotton (600TPI), } 1 \text { layer and } \\
\text { Silk, } 2 \text { layers }\end{array}$ & $94 \pm 2$ & $98.5 \pm 0.2$ \\
\hline $\begin{array}{l}\text { Cotton (600TPI), } 1 \text { layer and } \\
\text { Flannel, } 2 \text { layers }\end{array}$ & $95 \pm 2$ & 961 \\
\hline
\end{tabular}

TABLE I

SUMMARY OF FILTRATION EFFICIENCIES FOR A RANGE OF MATERIAL FROM PAPER B [27].

Grant, and Supratik Guha, 2020.

2) Overview: This paper directly measured the filtering efficiency of a large range of materials. A summary of their useful results at the lower flow rate is in table I] they had similar results for their higher flow rate test.

These results concur with the results from paper A in the cases where they overlap. This paper also discusses the mechanisms of filtration stating that some of the materials filter mechanically and some with electrostatic interactions resulting in higher filtration efficiencies when combined with multiple layers of the same material.

The paper also had results for a cotton quilt constructed from two cotton sheets filled with a $90 \%$ cotton, $5 \%$ polyester and $5 \%$ other fibers batting, this provided a filtration efficiency of $96 \pm 2 \%$ over the whole size range.

3) Discussion and take away: As the paper concludes, tight weave materials provide a good filtration efficiency, as do some materials which utilise electrostatic capture methods (silk, chiffon, flannel). Additionally when combined together to utilise both mechanical and electrostatic capture very high efficiency masks can be created.

The paper emphasises that their results suggest that very easily obtainable materials can provide a good filtration efficiency across the whole range, their emphasis was particularly on 4 layers of silk which can be found in many clothing items (e.g. scarfs) and the combinations which could easily be obtained from household items such as bedding (cotton) and sports wear (chiffon). It is particularly note worthy that their combinations and multiple layer tests performed better than the commercial N95 mask.

The paper also notes that the fit of the mask is a key factor in determining its filtration efficiency and a poor fit can reduce the filtration by more than $50 \%$.

C. Simple Respiratory Protection - Evaluation of the Filtration Performance of Cloth Masks and Common Fabric Materials Against 20 - 1000 nm Size Particles[28]

1) Authors: Samy Rengasamy, Benjamin Eimer and Ronald E. Shaffer, 2010.

2) Overview: This paper directly tested a very limited range of materials. In all cases across their range of cloth masks, sweatshirts, t-shirts, scarfs and towels which were made from mixes of cotton and polyester all had a penetration of $>50 \%$.

3) Discussion and take away: Little can be drawn from this paper when searching for good materials to use. It does highlight the fact that commercially available cloth masks/bandanas are likely to provide little to no protection to the user.

D. Expedient methods of respiratory protection. II. Leakage tests. Final report[29]

1) Authors: Douglas W. Cooper, William C. Hinds, John M. Price, Robert weker and Howell S. Yee, 1983

2) Overview: This paper conducted a thorough investigation of a range of materials in a range of conditions. Since this part of the review is primarily focused on the filtration abilities of materials we shall only consider the material elements not the methods by which they were attached to the model, it should be acknowledged that the paper does establish the fit of the mask is very important.

The papers most effective methods for attaching the materials was by taping around the whole sample or by using a nylon "panty hose" (or tights as they are better known in the UK) to hold the sample onto the model. As the tights do not provide any substantial increase in filtration over fully taping they only show another method for 


\begin{tabular}{|c|c|}
\hline Material & Filtration efficiency \\
\hline N95 reference & $98.5 \pm 0.24$ \\
\hline Surgical mask & $95.8 \pm 0.8$ \\
\hline $\begin{array}{l}\text { Shirt }(65 \% \text { polyester, } 35 \% \text { cotton, } 46 \\
\text { TPI) - } 4 \text { layers }\end{array}$ & $70 \pm \mathrm{pm} 1$ \\
\hline $\begin{array}{l}\text { Washcloth (terry weave } 88 \% \text { cotton, } \\
12 \% \text { Dacron/polyester) - } 1 \text { layer }\end{array}$ & $61 \pm 1$ \\
\hline $\begin{array}{l}\text { Handkerchief ( } 100 \% \text { cotton, 62TPI) - } 4 \\
\text { layers }\end{array}$ & $76 \pm 3$ \\
\hline
\end{tabular}

TABLE II

SUMMARY OF FILTRATION EFFICIENCIES FOR A RANGE OF MATERIAL FROM PAPER D [29]

successfully sealing fabric to the face. A summary of the useful results from the paper is in table II

3) Discussion and takeaway: These results are similar to the other papers on the filtration efficiency of cotton supporting its use in masks. The testing of the nylon hosiery in the paper was interesting in that it showed the efficiency of the seal, it did not however constitute further evidence for using a synthetic layer as well as cotton, this is likely because it was far too thin and ineffective a filter on its own (2\%). It should not be considered evidence that multiple layers do not work either.

Another interesting take away is that surgical masks provide a good level of protection when securely attached to the wearers face. This could be used in conjunction with some of the designs discussed below.

\section{E. Emergency Respiratory Protection Against Ra- diological And Biological Aerosols[30]}

1) Authors: Guyton, H G, Decker, H M and Anton, G T, 1959

\section{F. Overview}

A full copy of this paper could not be located and so it is of limited help although some information can be drawn from the abstract. The paper tested 18 materials and found 3 to be of sufficiently high efficiency (85\%) and low enough impedance to be used. These materials were 3 layers of toilet paper, 2 layers of bath towel and a cotton handkerchief folded to 8 layers thick. They also found that when the items were wet they were less efficient filters and significantly higher impedance.
1) Discussion and take away: Although of limited use this paper again supports the use of cotton in masks. It is also helpful to know that being wet degrades the filtration abilities of the materials

G. Selection of homemade mask materials for preventing transmission of COVID-19: a laboratory study [31]

1) Authors: Dijia Wang, Yanjun You, Xiaoli Zhou, Zhiyong Zong, Hao Huang, Hui Zhang, Xin Yong1, Yifan Cheng, Liu Yang, Qiong Guo, Youlin Long, Yan Liu, Jin Huang and Liang Du, 2020

2) Overview: This paper directly measured the filtration efficiencies and pressure drop of a range of materials. The aim of the paper was to find materials that filter to the Chinese standards for surgical masks [32] and so therefore they did not pursue higher efficiencies. They also had the luxury of medical fabrics and so base much of their findings on the availability of it. The paper did not find any materials with comparable filtering efficiencies to any of the other studies except with the medical materials.

3) Discussion and take away: This paper does not seem to be very thorough in trying to find materials that are effective filters. This shows that not all materials are effective filters but little else. The study used many materials that could be expected from the previous papers to be effective filters such as t-shirts, tea towels and pillow cases. It is therefore important to remember that high quality materials which the composition of which can be verified need to be used when looking for a higher efficiency mask.

\section{H. Others}

There are many un-published sources suggesting that other materials may be effective filters. There are too many to fully discuss but a few ideas should be mentioned.

1) Polypropylene bags: Some sources support the use of polypropylene bags as filtering materials on the basis that polypropylene is the material used in many commercial masks [33][34]. While it may be the case, there is no evidence to be found that polypropylene bags have a good filtration efficiency. There are many different manufacturing and weaving processes for materials with the same composition and so the use of the chemical in 
commercial masks provides no evidence for their filtering efficiency. On the other hand, lack of evidence is not evidence for inefficiency.

2) Halyard H600: There are several sources that advocate for the use of Halyard H600, a material used for wrapping and sterilising surgical instruments, for masks. There is some evidence that it is effective as masks have been made and then fit tested [35][36]. There are no direct tests of the materials. This isn't a particularly useful finding since it is hard or impossible to get this material.

3) Air conditioning filters: While less common in the UK, in the United States there has been a some what wide spread use of air conditioner filters for masks [37][38]. Air filters are held to a legal standard and so should provide a high filtration efficiency ( $>99.95 \%$ for $\mathrm{H} 13$ and above) [39]. While there is little published testing of HEPA filters used in masks there creator of one of the following designs informed me that in their as yet unpublished testing they found a filtration efficiency of $98 \%$ when using 2 layers of " $3 \mathrm{M}$ Filtrete MPR 2200"[40]. One limitation of this material could be the difficulty in removing it cleanly from the filter and it being quite stiff, which could be more useful for the ridged designs below.

\section{DESIGNS}

As we have seen from the review of these papers a key factor in the efficacy of masks is dependent on how well the mask fits on the users face. In an ideal scenario every person needing a mask would have access to a fit testing method as this would determine on an individual basis whether the design was suitable. Since this is clearly impossible a reasonable method to determine the suitability of a design could be to look at fit test results conducted on other people. Although of limited use this seems to be the only reasonable method. It is by no means certain that a good fit on one person would be good on another.

In the current climate there are a huge number of designs for masks which could not all be analysed so a small selection are listed here. There are essentially 2 broad categories of masks: fabric and ridged designs.

\section{A. Fabric designs}

1) Weibel Surgical Mask[41]: This design is a simple folded design as shown in 1 . The mask was made from Halyard H600 and so was heat sealed around the edges making it only some what useful in a home made setting.

Fig. 1. Weibel surgical mask. Photo by Thomas Sharkey, reproduced with permission.

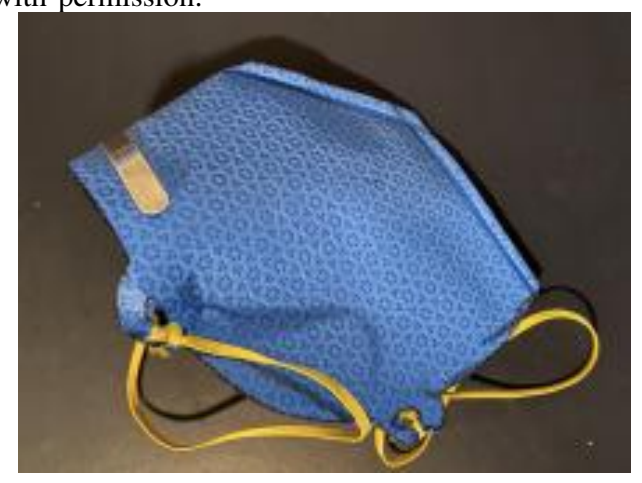

In their fit testing they achieved approximately 98.5\% filtration, greater than their N95 control ( $95 \%$ filtration). They also passed fit tests, in come cases with better fit factors than their control N95 mask [42]. The pattern could be used as a guide for sewn masks, it should be noted that they had an aluminium strip across the nose, this is a common theme with the effective designs.

2) Suay Community Mask Coalition[43]: This mask is a flatter design and is fully sewn. In their fit testing they achieved an $82 \%$ filtration, this shows that the mask fits well. Their advocated materials which are possibly less effective filters so the fit could possibly be better than this with improved materials.

3) HKMask [44]: This mask appears to be better researched. It is of a much simpler design with inner layers trapped against the face. In their fit testing they achieved fits of up to $91 \%$ with multiple filter layers. This suggests that it provides a good fit which is mainly material dependent. It notably doesn't include a nose wire, possibly making it easier to make.

4) University of Florida Health [45]: This mask is again made of Halyard although these designs are sewn so are applicable to other materials. There is no record of the exact results of the fit tests conducted on these masks merely a statement that they passed, they are very similar to the Weibel 
Fig. 2. HKMask. Photo Dr. K Kwong, with MIT licence.

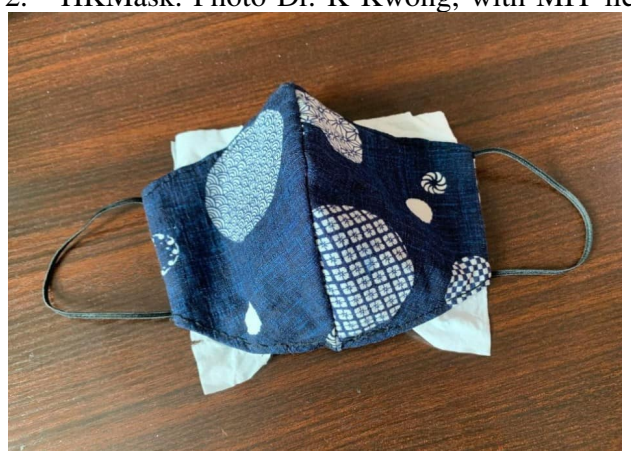

design. Since the pass standard is for an N95 mask it should be reasonable to assume that they achieved $95 \%$ filtration efficiency.

5) Tommye Austin[46]: This design is a duckbill design which is fully sewn. Media reporting of the design claims that it can provide $99.5 \%$ filtration efficiency but the only testing is a statement that it passed a fit test, as with the University of Florida Health design.

\section{B. Rigid designs}

The multitude of rigid deigns are largely 3D printed. This makes them some what less accessible for people to produce although it has been shown in recent times that the $3 \mathrm{D}$ printing community it good at distributed mass production to the scale that they could equip whole communities with PPE[47][48].

1) The Montana Mask[49]: This mask is rather more complicated than the fabric designs as can be seen in figure 3. It also takes a significant amount of time to produce. In their testing they achieved a fit factor of $200+$ so provides $99.5 \%$ filtration [50]. The filtering material used was a small piece of an $\mathrm{N} 95$ respirator.

2) Sani-Mask[51]: Sani-Mask is an interesting design because, although the fit was not tested the author claims that it fits well. This masks inclusion in this review is not for the possible fit but to raise the issue of cleaning of $3 \mathrm{D}$ printed masks. Research by the creator of this mask shows that it very hard to clean $3 \mathrm{D}$ printed masks, even if it can be shown when they are swabbed, due to the nature of 3D printed products and the fact that they usually contain hollow cavities.

This mask utilises a special 3D printing mode which removes this problem as it is single walled,
Fig. 3. The Montana Mask. Photo by Make The Mask, reproduced with permission.

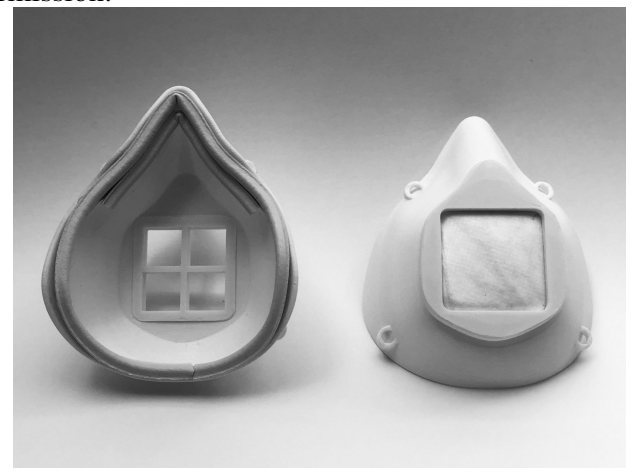

Fig. 4. Sani-Mask. Photo by Nemotech, reproduced with permission.

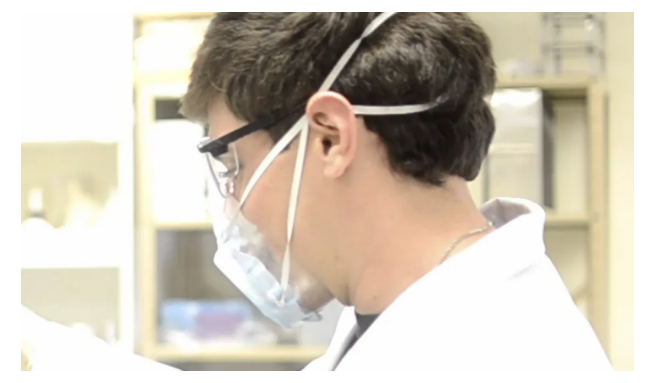

as can be seen in figure 4 . Another benefit to this is that it takes significantly less time to print than other designs.

3) Wiles Covid Pandemic Mask[52]: This design is quite similar to The Montana Mask but has better documented testing and continued development, including extensive YouTube videos on their construction [53]. The documentation notes that it has passed fit tests for many people with a variety of face shapes, particularly when modified to have sealing foam stuck to the inside.

The creator also recommends spraying the outside with sealant, this reduces the likely hood of the tiny cracks that are usually present in 3D printed items from leaking and prevents the outside from absorbing liquids. The design also recommends gluing the filter in with hot glue to glue the parts together. From the documentation of this mask it seems likely that it could prove to be a much more reliable design that will fit a range of people. It is helpful that it has been used by and tested on so many people to support this assumption.

The sealing is an interesting point that should be consider with all 3D printed designs. 
4) Altavian / American Mask Rally Response Mask [54]: This mask is also similar to The Montana Mask. It distinguishes it self in that it has a much larger filter area, likely to make it more usable, and that it has less material and is so likely to take less time to make. It is similarly limited by the requirement for more materials than most people have available.

The creator states that this mask has passed several fit tests ( $99 \%$ filtration efficiency).

\section{Frame designs}

There are several very similar frame designs which essentially work by providing a solid ring which pins fabric against the face of the user by attachment points for elastic as can be seen in figure 5[55][56][57].

Fig. 5. Frame type masks. Photo by Dr Chris Wiles, reproduced with permission.

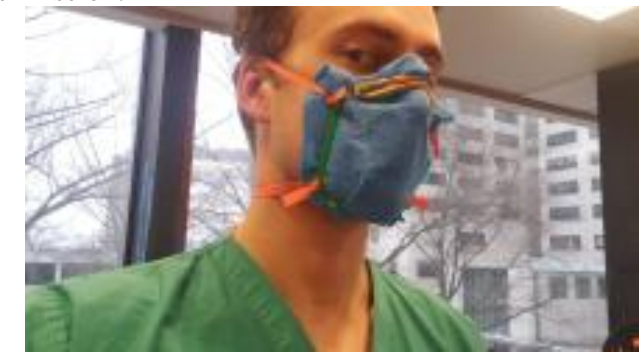

As far as could be seen none of the frame designs have passed fit tests, likely because they are being used in communities more than in health care settings where more substantial designs are being used. Several of the authors do mention good fits but none are verified. These designs have the advantage that they take significantly less time to produce than other ridged designs and also require much less skill to construct than sewn designs.

\section{Hygiene, CLEANing AND REUSE}

It is key that masks are used as part of a wider hygiene system [58] including hand hygiene and disinfecting objects that are potentially contaminated. It is therefore key that any mask that is used is properly cleaned before it is introduced back into a safe place such as the users home of office. Some methods of decontamination which should be accessible in the home setting are listed below. This is a vast subject in its self but will be made brief in this review.
In reviews by Stanford Medicine [59] and by a group called N95Decon composed of academics from across the United States [60] both find that heating in at 70 to $85^{\circ} \mathrm{C}$ with a greater than $50 \%$ humidity for 60 minutes in an effective method for decontaminating masks from SARS-CoV-2. This can be replicated at home[61]. They also stress that decontamination methods vary by pathogen.

An alternative method, though somewhat less well researched as not applicable to any healthcare setting or commercial masks, is machine washing. It is likely that for this to be an effective method strong detergents such as bleach are required as well as a long drying cycle [62], this could remove up to $99.99 \%$ of viruses in the wash. The $\mathrm{CDC}$ also recommends washing at $>70^{\circ} \mathrm{C}$ for 25 minutes or for materials which can not tolerate higher temperatures using a suitable disinfectant (e.g. bleach)[63]. These recommendations should be taken into account when choosing materials as some may not tolerate temperature or disinfectants.

These cleaning methods are clearly unsuitable for the rigid components of some of the mask designs. As mentioned when discussing the SaniMask it is hard to clean some plastic parts due to their permeability. This is a key area for more research as a large amount of research in the area focuses on swabbing of the surface which may not be suitable [51]. Hot air drying is likely to also be suitable for plastic components as well as cleaning in harsher chemicals such as iso-propanol or bleach [64]. It should be noted that the glass transition temperature of the plastics needs to be considered when choosing the plastic for the mask if it is to be hot air sterilised.

A last resort methods of decontamination could be to leave the mask in a warm, moderately humid place for 5 to 7 days [65].

A final key point in the use of masks is their donning and doffing. Many organisations provide guidelines for this which should be reviewed.

A key theme among many guidelines from the NHS and the CDC can be summarised as below [66][67][68][69].

Putting the mask on (donning):

1) The user should sanitise their hands before and after touching the mask.

2) The mask should be put on before other 
protection (e.g. gloves and eye protection).

3) The mask should be held with the cup of the mask in one of the users hand and pressed against the face, the other hand should then be used to place straps over the users head.

4) Once the mask is on the user should mould the mask around the nose.

Taking the mask off (doffing):

1) The mask should be the last item of PPE to be removed - in a community setting this could include clothes worn in a potentially contaminating environment.

2) The user should sanitise their hands before and after touching the mask.

3) The mask should be touched as little as possible, it should be held only by the straps and placed in a sealed container as efficiently as possible to be cleaned.

\section{FIT AND FIT TESTING}

It has become clear that the fit of masks is key. As previously stated masks, as with commercial masks, should be fit tested for users on an individual basis. There has been some research into the suitability of home made fit testing solution [70] which finds it to be as effective as commercial solution (also of note they find that qualitative fit testing is a bad indicator of fit). The problem with this though is that it still relies on the availability of the nebuliser and hood used for normal testing. These could be improvised but further investigation is required.

There are CDC guidelines for checking the seal of respirators [71] by feeling for pressure changes when the mask the user breathes in or out in restricted manners. It is stressed that this should only be done with masks that the user has previously had a full fit test for although it could be useful when no other options are available.

\section{CONCLUSIONS AND RECOMMENDATIONS}

In conclusion; there is substantial evidence that high thread count cotton provides a moderate filtration efficiency against particles of a similar size to SARS-CoV-2 at around $70 \%$. There is some evidence that adding multiple layers of other materials such as chiffon, silk or flannel increases this efficiency to the same order as commercially available masks (95\%). There is also some evidence that other materials such as vacuum cleaner bags can provide a similar filtration efficiency. In order to improve the confidence in the higher filtration material combinations more supporting research needs to be conducted to directly measure the efficiencies, the most helpful choices for further research would be chiffon, silk and flannel as well as a wide range of named brands of vacuum cleaner bags. When they are available the material surgical masks are made from are likely to be effective filters, they are only floored in their seal to the users face which could be improved by many of the designs.

The fit of the mask is also found to be key to its efficiency and also an area substantially lacking exploration. The only recommendation that can be made is that masks should be fit tested on an individual basis but failing that, the user should use their judgement and feel of the pressure in the mask (although this provides very little certainty of a good fit). It appears that there are designs that have potential, both fabric and rigid. It would be helpful for an alternative fit testing process to be devised with house hold items, this is unlikely to be created since the qualitative fit testing process is sometimes deemed to be a poor indicator of fit[70]. Fit testing could be provided as a service to the public in order to increase the work forces ability to return to normal.

\section{REFERENCES}

[1] MacIntyre CR, Zhang Y, Chughtai AA, Seale H, Zhang D, $\mathrm{Chu} \mathrm{Y}$, et al. Cluster randomised controlled trial to examine medical mask use as source control for people with respiratory illness. BMJ Open. 2016;6(12). Available from: https: //bmjopen.bmj.com/content/6/12/e012330

[2] van der Sande M, Teunis P, Sabel R. Professional and Home-Made Face Masks Reduce Exposure to Respiratory Infections among the General Population. PLOS ONE. 2008 07;3(7):1-6. Available from: https://doi.org/ 10.1371/journal.pone.0002618

[3] Sung AD, Sung JAM, Thomas S, Hyslop T, Gasparetto C, Long G, et al. Universal Mask Usage for Reduction of Respiratory Viral Infections After Stem Cell Transplant: A Prospective Trial. Clinical Infectious Diseases. 2016 08;63(8):999-1006. Available from: https://doi.org/ $10.1093 / \mathrm{cid} / \mathrm{ciw} 451$

[4] Greenhalgh T, Schmid MB, Czypionka T, Bassler D, Gruer L. Face masks for the public during the covid-19 crisis. BMJ. 2020;369. Available from: https://www.bmj.com/ content/369/bmj.m1435

[5] Leung NHL, Chu DKW, Shiu EYC, Chan KH, Mcdevitt JJ, Hau BJP, et al. Respiratory virus shedding in exhaled breath and efficacy of face masks. Nature Medicine. 2020 Mar; 
[6] Dharmadhikari AS, Mphahlele M, Stoltz A, Venter K, Mathebula R, Masotla T, et al. Surgical Face Masks Worn by Patients with Multidrug-Resistant Tuberculosis. American Journal of Respiratory and Critical Care Medicine. 2012;185(10):1104-1109.

[7] Tracht SM, Valle SYD, Hyman JM. Mathematical Modeling of the Effectiveness of Facemasks in Reducing the Spread of Novel Influenza A (H1N1). PLoS ONE. 2010 Oct;5(2).

[8] Offeddu V, Yung CF, Low MSF, Tam CC. Effectiveness of Masks and Respirators Against Respiratory Infections in Healthcare Workers: A Systematic Review and Meta-Analysis. Clinical Infectious Diseases. 2017 Jul;65(11):1934-1942.

[9] Garcia Godoy LR, Jones AE, Anderson TN, Fisher CL, Seeley KML, Beeson EA, et al. Facial protection for healthcare workers during pandemics: a scoping review. BMJ Global Health. 2020;5(5). Available from: https://gh.bmj.com/ content/5/5/e002553

[10] Greenhalgh T. Face coverings for the public: Laying straw men to rest. Journal of Evaluation in Clinical Practice;n/a(n/a):e13415. Available from: https://onlinelibrary.wiley.com/doi/abs/ 10.1111/jep.13415.

[11] of Health D, Care S. Coronavirus (COVID-19): personal protective equipment (PPE) plan. GOV.UK; 2020. Available from: https://www.gov.uk/government/ publications/coronavirus-covid-19personal-protective-equipment-ppe-plan

[12] Swaminathan A, Martin R, Gamon S, Aboltins C, Athan E, Braitberg G, et al.. Personal protective equipment and antiviral drug use during hospitalization for suspected avian or pandemic influenza. Centers for Disease Control and Prevention; 2007. Available from: https://www.ncbi.nlm.nih.gov/pmc/articles/ PMC2851524/

[13] Immediate management of a suspected case of $\mathrm{CoV}$ 2019(Wuhan) or Avian influenza for acute sites GGI\&C. NHS Greater Glasgow and Clyde; 2020. Available from: https: //www.nhsggc.org.uk/media/258206/airbornehcid-guidance-ggc-v4-final-2801.pdf

[14] EN 149 - Respiratory protective devices- Filtering half masks to protect against particles - Requirments, testing, marking. BSI; 2009.

[15] CDC - NIOSH-Approved N95 Particulate Filtering Facepiece Respirators - 3M Suppliers List. Centers for Disease Control and Prevention; 2020. Available from: https://www.cdc.gov/niosh/npptl/topics/ respirators/disp_part/n95list1.html

[16] HEALTH GB, EXECUTIVE S. GUIDANCE ON RESPIRATORY PROTECTIVE EQUIPMENT (RPE)FIT TESTING: (pack of 5). HSE BOOKS; 2019.

[17] Macintyre CR, Cauchemez S, Dwyer DE, Seale H, Cheung P, Browne G, et al. Face Mask Use and Control of Respiratory Virus Transmission in Households. Emerging Infectious Diseases. 2009;15(2):233-241.

[18] Davies A, Thompson KA, Giri K, Kafatos G, Walker J, Bennett A. Testing the Efficacy of Homemade Masks: Would They Protect in an Influenza Pandemic? Disaster medicine and public health preparedness. 2013 08;7:413-418.

[19] 6 or 12 Pack Big Check Tea Towel Cotton Absorbent, Black, 6 Pack; Available from: https: //www.amazon.co.uk/dp/B086DXMJY7/ref= Cm_SW_r_tw_dp_U_X_sW2ZEbEMNKQK
[20] PORTER AND LAMBERT Terry Tea Towel - 100\% Egyptian Cotton: Professional Grade: Essential Dish Cloths for Kitchen/Restaurant/Bar Glass: Pack of 6 I\& 12 - $(6, \quad 75$ x $50 \quad \mathrm{~cm}) ; . \quad$ Available from: https://www.amazon.co.uk/dp/B084 JMHQMN/ref= cm_SW_r_tw_dp_U_X_fY2ZEbXXDJFGN

[21] Utopia Towels - 12 Kitchen Towels Set - 38 x 64 cm, Black and White - $100 \%$ Ring Spun Cotton Super Soft and Absorbent Dish Towels, Tea Towels and Bar Towels;. Available from: https://www.amazon.co.uk/dp/B009N8301C/ $r e f=c_{1}$ _Sw_r_tw_dp_U_x_UY2ZEbV97BGSE

[22] AmazonBasics Sateen Pillowcase - $50 \quad$ x $80 \quad \mathrm{~cm} \quad x$ 2, Black: Amazon.co.uk: Kitchen I\& Home;. Available from: https://amazon.co.uk/dp/B07H3S5NKS/ $r e f=m_{-}$sw_r_tw_dp_U_x_sZ2ZEbA7AY 914

[23] RAYYAN LINEN WHITE 100\% EGYPTIAN COTTON PAIR OF PILLOWCASES 200 THREAD COUNT 50 X 75 CM: Amazon.co.uk: Kitchen I\& Home;. Available from: https://amazon.co.uk/dp/B00HAN52GC/

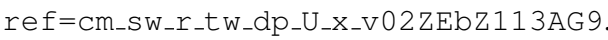

[24] Willeke K, Trakumas S, Grinshpun SA, Reponen T, Trunov M, Friedman W. Test Methods for Evaluating the Filtration and Particulate Emission Characteristics of Vacuum Cleaners. AIHAJ - American Industrial Hygiene Association. 2001;62(3):313-321. Available from: https://doi.org/ $10.1080 / 15298660108984633$

[25] Hoover Purehepa Vacuum Cleaner Bags I\& Filter H63: Amazon.co.uk: Kitchen I\& Home;. Available from: https://amazon.co.uk/dp/B00134BRPO/ref= Cm_Sw_r_tw_dp_U_x_W12ZEb9AEDDR1

[26] Miele Vacuum Cleaner Dust Bags, Microfibre Blend, 1-Pack, A: Amazon.co.uk: Kitchen I\& Home;. Available from: https://amazon.co.uk/Miele-Vacuum-CleanerMicrofibre-1-Pack/dp/B00B8RT4FG/ref= Sr_1_7

[27] Konda A, Prakash A, Moss GA, Schmoldt M, Grant GD, Guha S. Aerosol Filtration Efficiency of Common Fabrics Used in Respiratory Cloth Masks. ACS Nano. 0;0(0):null. PMID: 32329337. Available from: https://doi.org/ $10.1021 /$ acsnano.0c03252

[28] Rengasamy S, Eimer B, Shaffer RE. Simple Respiratory Protection-Evaluation of the Filtration Performance of Cloth Masks and Common Fabric Materials Against 20-1000 nm Size Particles. The Annals of Occupational Hygiene. 2010 06;54(7):789-798. Available from: https://doi.org/ 10.1093/annhyg/meq0 44

[29] Cooper DW, Hinds WC, Price JM, Weker R, Yee HS. Expedient methods of respiratory protection. II. Leakage tests. Final report. US Department of Energy Office of Scientific and Technical Information;

[30] Guyton HG, Decker HM, Anton GT. EMERGENCY RESPIRATORY PROTECTION AGAINST RADIOLOGICAL AND BIOLOGICAL AEROSOLS. AMA Arch Ind Health;.

[31] Wang D, You Y, Zhou X, Zong Z, Huang H, Zhang $\mathrm{H}$, et al. Selection of homemade mask materials for preventing transmission of COVID-19: a laboratory study. medRxiv. 2020;Available from: https://www.medrxiv.org/content/early/ 2020/05/11/2020.05.06.20093021

[32] Food C, Administration D. YY 0469-2011 Surgical mask; 2011. Available from: http://codeofchina.com/ standard/YY0469-2011.html

[33] Liz. DIY N95 Masks Medical Polypropylene Face Masks; 
2020. Available from: https://diysoldier.com/diyn95-medical-polypropylene-face-mask/

[34] Materials;. Available from: https://makermask.org// materials/

[35] Mask Alternative " Department of Anesthesiology " College of Medicine "University of Florida;. Available from: https://anest.ufl.edu/clinical-divisions/ mask-alternative/

[36] Buletti L. UF Health anesthesiology team devises respirator mask made from existing hospital materials; 2020. Available from: https://ufhealth.org/news/2020/ uf-health-anesthesiology-team-devisesrespirator-mask-made-existing-hospitalmaterials

[37] stlukessimcenter. 3d Mask - SLUHN and Filament Innovations. U.S. Department of Health and Human Services; 2020. Available from: https://3dprint.nih.gov/ discover/3dpx-013321

[38] Winder M. 3D Printable, Breathable Handkerchief Alternatives. U.S. Department of Health and Human Services; 2020. Available from: https://3dprint.nih.gov/ discover/3dpx-013384

[39] EN 1822-1 - High efficiency air filters (EPA, HEPA and ULPA). Classification, performance testing, marking. BSI; 2019.

[40] Wiles C. Wiles Covid Pandemic Mask Testing; 2020.

[41] Weibel Surgical Mask. U.S. Department of Health and Human Services;. Available from: https://3dprint.nih.gov/ discover/3dpx-014003

[42] Patel S, Sharkey T, Weibel N. WeibelLab / Triton-Mask / FitTesting; 2020. Available from: https://github.com/ WeibelLab/Triton-Mask/wiki/Fit-Testing

[43] SUAY COMMUNITY MASK COALITION;. Available from: https://suayla.com/pages/suay-communitymask-coalition

[44] HKMask Manual ;. Available from: https: //diymask.site/

[45] Mask Alternative " Department of Anesthesiology " College of Medicine "University of Florida;. Available from: https://anest.ufl.edu/clinical-divisions/ mask-alternative/

[46] Gray J, Galvan J. Nurse shares step-by-step instructions for making mask with up to $99.5 \%$ filtration efficiency. KSAT San Antonio; 2020. Available from: https://www.ksat.com/news/local/2020/04/ 16/local-nurse-designs-mask-with-995filtration-efficiency/

[47] 3D Crowd UK - 3D Printed Face Shields For Front Line Workers; 2020. Available from: https:// 3dcrowd.org.uk/

[48] Masks DDP. Doctors 3D Printing Masks organized by Christopher Wiles; $2020 . \quad$ Available from: https://www.gofundme.com/f/doctors-3dprinting-masks

[49] Make The Masks; //www.makethemasks.com/

[50] UNITED STATES DEPARTMENT OF LABOR;. Available from: https://www.osha.gov/pls/oshaweb/ owadisp.show_document?p_id=9780I\&p_table= STANDARDS

[51] Nemotech hcm. Sani-Mask;. Available from: https:// nemotech3d.com/sani-mask

[52] Wiles C. VERSION 2: Wiles COVID Pandemic Mask - Fight the PPE Shortage, by ctwiles; 2020. Available from: https: //www.thingiverse.com/thing:4237783/files

[53] Wiles C. ChrisWiles. YouTube;. Available from: https: //www.youtube.com/user/chrischris64

[54] Altavian / American Mask Rally Response Mask - Reusable, Changeable Filter. U.S. Department of Health and Human Services;. Available from: https://3dprint.nih.gov/ discover/3dpx-014278

[55] Wiles C. "CEG Extreme" COVID19 Mask Frame for Halyard H600. U.S. Department of Health and Human Services; 2020. Available from: https://3dprint.nih.gov/ discover/3dpx-013690

[56] Fuller M. GE Additive Community Face Mask Frame. U.S. Department of Health and Human Services; 2020. Available from: https://3dprint.nih.gov/discover/3dpx013512

[57] UD HensNest. U.S. Department of Health and Human Services;. Available from: https://3dprint.nih.gov/ discover/3dpx-013376

[58] Infection prevention and control during health care when novel coronavirus (nCoV) infection is suspected. World Health Organization;. Available from: https://www.who.int/publications-detail/ infection-prevention-and-control-duringhealth-care-when-novel-coronavirus(ncov)-infection-is-suspected-20200125

[59] Addressing COVID-19 Face Mask Shortages [v1.; 2020. Available from: https:// stanfordmedicine.app.box.com/v/covid19PPE-1-1

[60] Technical Report for Heat-Humidity-Based N95 Reuse Risk Management; $2020 . \quad$ Available from: https://staticl.squarespace.com/ static/5e8126f89327941b9453eeef/ t/5ea25d8b4965f32f414d974c/ $1587699083786 / 2020-04-$ 23_N95DECON_Heat_Technical_Report_v2.0_final.pdf

[61] Mechler S, MechlerScott S, Scott, Consolidated, University N. Covid-19 Pandemic: Disinfection and Sterilization of Face Masks for Viruses; 2020. Available from: https://consteril.com/covid-19-pandemicdisinfection-and-sterilization-of-facemasks-for-viruses/

[62] Gerba CP, Kennedy D. Enteric Virus Survival during Household Laundering and Impact of Disinfection with Sodium Hypochlorite. Applied and Environmental Microbiology. 2007;73(14):4425-4428. Available from: https: //aem.asm.org/content/73/14/4425.

[63] ; 2019. Available from: https:// www.cdc.gov/infectioncontrol/guidelines/ environmental/recommendations.html

[64] Prusa Face Shield disinfection;. Available from: https://help.prusa3d.com/en/article/prusaface-shield-disinfection_125457

[65] Technical Report for Time-Based N95 Reuse Risk Management; $2020 . \quad$ Available from: https://static1.squarespace.com/ static/5e8126f89327941b9453eeef/ t/5ea3b2ea36c5d45a8f2dd331/ $1587786474427 / 2020-04-$ 24_N95DECON_Time_Technical_Report_v1_final.pdf

[66] Ambulance Service PPE Donning I\& Doffing Guidance; 2020. Available from: https:// 
ntk.eastamb.nhs.uk/Documents/Instructions/

AmbulanceServicePPEDonningandDoffingGuidanceEEAST.pdf

[67] Putting on (donning) personal protective equipment (PPE)

for aerosol generating procedures (AGPs);. Available from:

https://assets.publishing.service.gov.uk/

government/uploads/system/uploads/

attachment_data/file/879103/PHE_COVID-

19_Donning_quick_guide_gown_version.pdf

[68] Removal of (doffing) personal protective equipment (PPE) for aerosol generating procedures (AGPs);. Available from: https://assets.publishing.service.gov.uk/ government/uploads/system/uploads/ attachment_data/file/879104/PHE_COVID-

19_Doffing_quick_guide_gown_version.pdf

[69] How to Properly Put on and Take off a Disposable Respirator;. Available from: https://www.cdc.gov/niosh/docs/ 2010-133/pdfs/2010-133.pdf

[70] Fakherpour A, Jahangiri M, Yousefinejad S, Seif M. Feasibility of replacing homemade solutions by commercial products for qualitative fit testing of particulate respirators: a mixed effect logistic regression study. MethodsX. 2019;6:1313-1322.

[71] User Seal Check;. Available from: https: //www.cdc.gov/niosh/docs/2018-130/pdfs / 2018-130.pdf? id=10.26616/NIOSHPUB2018130 